\title{
Optimal Energy Window Selection for Scintigraphy \& Emission Computed Tomography
}

\author{
Kevin Wells, Afidalina Tumian, Angelos Zapros, Mohammed Alnafea, \\ M. Iqbal Saripan, Matthew Guy \& Paul Hinton
}

\begin{abstract}
Radioisotope imaging methods such as PET, SPECT and planar scintigraphy, currently utilize a fixed energy (pulse height) acceptance window regardless of the volume of the subject being imaged. This is despite previous work suggesting that higher window settings may yield improved image quality for imaging larger objects. However, we speculate that non-standard energy windows have not been widely adopted because there has not, until now, been a method available for determining how and when to use such an approach. In this new work we address this issue and propose a method for setting an adaptive photopeak acceptance window. This should be optimal for a wide variety of imaging situations applied across different radioisotope imaging methods. In order to develop an automatic technique in which individual patient-specific optimal thresholds can be determined, we propose a Bayes' Minimum Error Thresholding approach, which utilizes modeling the upper part of the observed energy spectrum as a two-class Gaussian mixture model. Exemplar planar Monte Carlo results and a preliminary phantom study are presented.
\end{abstract}

\section{INTRODUCTION}

$\mathrm{T}_{\mathrm{s}}$ HERE has been a long history of work published on threshold selection for defining energy windows around photopeaks and other structure in radionuclide spectra [e.g.1-3]. In the particular case of imaging Tc-99m radio nuclide distributions with a gamma camera, the accepted energy window of 15 or $20 \%$ about the photopeak is used as an accepted trade-off between minimizing the acceptance of scattered photons, and maximizing the acceptance of true unscattered radiation (sensitivity). In the case of PET imaging, a similar energy window is defined dependent on the scintillation crystal used.

A number of publications describe the use of asymmetric windows; work by Koral [4] reported that the use of an energy window shifted upwards to produce a $20 \%$ reduction in counts could yield improvements in image quality and in image quantification: error in absolute quantification of hot $0.6 \mathrm{~cm}^{3}$ insert in a phantom was reduced from $20 \%$ to $3 \%$.

Similarly, Graham et al [5] concluded that an asymmetric

Kevin Wells*, Angelos Zapros, Mohammed Alnafea and M.Iqbal Saripan are with the School of Electronics \& Physical Sciences, University of Surrey, Guildford, Surrey GU2 5XH, UK.

Afidalina Tumian is now with the Department of Electrical \& Computer Engineerng, Kulliyyah University, P.O. Box 10, 50728 Kuala Lumpa, Malaysia.

Matthew Guy and Paul Hinton are with the Nuclear Medicine Department, Royal Surrey County Hospital NHS Trust, Guildford, GU2 7XX. window in the presence of scatter could improve spatial resolution by $20 \%$ at FWTM, with improved observer defined contrast, but made the important distinction that most of the improvements in image quality could be obtained by simply shifting the energy window to produce a $10 \%$ reduction in counts compared to a window centered on $140 \mathrm{keV}$.

More recently, Turkington et al [6,7] suggested the use of an asymmetric window about the photopeak in PET wherein, instead of shifting the window, only the lower energy threshold was raised for imaging large objects.

However, despite the apparent advantages, the use of these non-standard energy windows has failed to find popular acceptance. This may be due to historical gamma camera susceptibility to drift in PMT gain, so that precise thresholds could not be previously be maintained, and that shifting the energy window may have produced flat field non-uniformity problems. The first two points may no longer be such a major concern with the advent of modern digital systems. However, of more significant importance is the observation made by Buvat et al [8] that there is no optimal fixed shift that can apply for all imaging situations, since the shift of the window is dependent on a large set of factors.

We attempt to address this situation in the work presented below. First we describe the application of a 2-component Gaussian mixture model to the upper portion of the observed energy spectrum. Then we consider parameter estimation and fitting via three spectral energy windows to solve a set of simultaneous equations. The estimation of the Bayes' Minimum Error threshold is then described. We illustrate the approach by using Monte Carlo simulation to examine the change in threshold for a simple imaging geometry and then consider its application to a preliminary phantom experiment.

\section{SPECTRUM MODELLING}

\section{A. Application of a Gaussian Mixture Model}

According to Berger and Seltzer [9], the energy response of a gamma camera can be modeled as:

$$
R\left(E_{o}, h\right)=\eta\left(E_{0}\right) \int_{0}^{E_{0}} D\left(E_{0}, E\right) \cdot G(E, h) d E
$$

where $R\left(E_{0}, h\right) d h$ represents the probability that a gamma ray, incident with energy $E_{0}$, will give rise to a pulse with a height between $h$ and $h+d h . \eta\left(E_{o}\right)$ is the detection efficiency, which represents the probability that the incident gamma ray, with energy $E_{0}$, will have at least one interaction in the detector. $D\left(E_{0}, E\right) d E$ is the energy deposition distribution, which represents the probability that, given the gamma ray interacts with the detector with energy $E_{0}$, it will deposit an amount of 
energy between $E$ and $E+d E . G(E, h) d h$ is the resolution function, which represents the probability that the deposition of energy $E$, will give rise to a pulse with a height between $h$ and $h+d h$.

Equation 1 involves the convolution between $D\left(E_{0}, E\right)$ and $G(E, h)$. However, by ignoring scattering events, and considering only photoelectric interactions in the scintillation crystal produced by a mono-energetic photon source with energy $E_{p}$, then equation 1 reduces to

$$
R\left(E_{p}, h\right)=\eta\left(E_{p}\right) D\left(E_{p}, E_{p}\right) \cdot G\left(E_{p}, h\right)
$$

where $D\left(E_{p}, E_{p}\right)$ is a constant, proportional to the photoelectric cross-section at energy $E_{p}$, and $G\left(E_{p}, h\right)$ is modeled as a Gaussian distribution representing the statistical variation in photoelectron signal produced in the PMT array.

Consider now the shape of the Compton edge: this will represent a convolution of non-stationary Gaussian kernels of energies in the interval 0 to $E<E_{p} . \mathrm{keV}$. We now heuristically suggest that this edge can be approximated, over a limited range, as a single Gaussian, with an empirically defined width $\sigma_{C}$ and a peak $\mu_{C}$ slightly less that $E_{p}$. So for Compton events with energy in the range $\left(\mu_{C}, E_{P}\right)$ this can be expressed as:

$$
R\left(E_{C}, h\right) \cong \eta\left(E_{C}\right) G(E, h)
$$

where $\eta\left(E_{C}\right)$ represents a single scaling factor, and $G^{\prime}(E, h)$ represents an empirically defined Gaussian function.

The observed spectrum is then simply the sum of equations 2 and 3 , scaled by $w\left(E_{i}\right)$, the prior probability of a particular photon with energy $E_{i}$ being incident on the detector. i.e. representing the relative photon flux at each energy, in the interval $\left(0, E_{p}\right) . w\left(E_{i}\right)$ is dependent on the pathlength and object densities encountered by the photon. This can be decomposed into a resulting prior probability for an unscattered photon to be incident on the detector $w\left(E_{P}\right)$, and that for a Compton scattered photon to be incident, $w\left(E_{C}\right)$. So the upper portion of the observed differential pulse height spectrum can be written as a scaled 2-class Gaussian mixture model:

$$
R\left(E_{C}, h\right) \cong w\left(E_{C}\right) \eta\left(E_{C}\right) G(E, h)+w\left(E_{P}\right) \eta\left(E_{P}\right) G(E, h)
$$

\section{B. Bayes' Minimum Error Threshold}

The Bayes' Minimum Error Threshold is a classic method used in pattern recognition for estimating group memberships in the face of uncertain evidence [14]. There are various formulations, but that used here has been developed for 1-D data containing Gaussian noise. In this section we summaries the main aspects with respect to optimal thresholding in nuclear medicine.

A definition of the optimal energy threshold is necessary before proceeding. Thus, in the context of radioisotope imaging, we define the optimal energy threshold as the lower energy threshold of the detector system's acceptance window that minimizes the misclassification of scatter events as photopeak detections whilst maximizing detection of the true photopeak events. Figure 1 illustrates an example optimal threshold with the two Gaussians fitted to describe the photopeak and Compton edge. Note that if the optimal threshold shifts to the left (lower energy) then any gain in full absorption (photopeak) event detection is offset by a greater increase in scatter detection. If the threshold shifts to the right, then photopeak efficiency is diminished to a greater extent than the desired suppression of scatter.

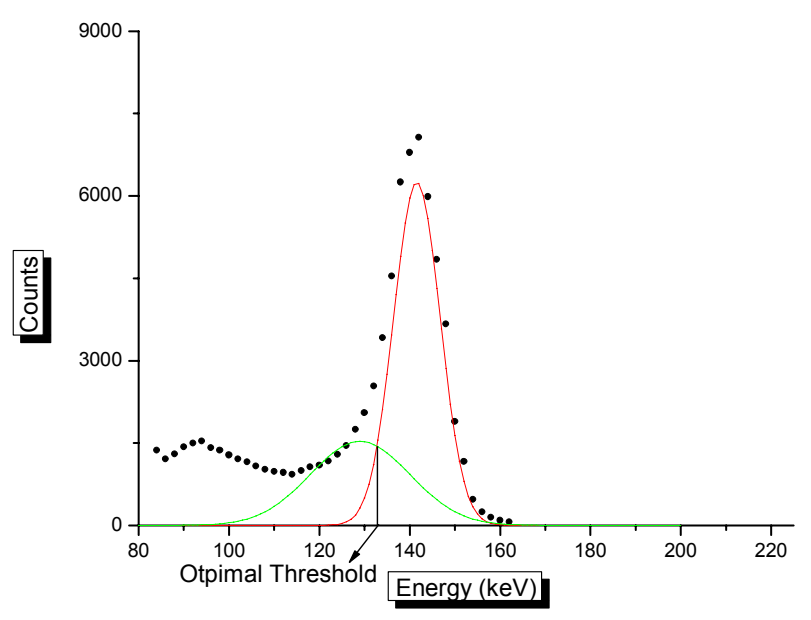

Figure 1: Example of the two Gaussians fitted to describe the photopeak and Compton edge.

Given the fraction of spectral events in the Compton scattered part of the spectrum is $\gamma_{\mathbf{S}}$ and that the scatter pulse height distribution is described by a pdf, $S(h)$, then the proportion of misclassified scatter detections within the photopeak window above some thereshold, $T$, will be given by:

$$
\varepsilon_{S}=\gamma_{S} \int_{T}^{+\infty} S(h) d h
$$

Similarly, if the fraction of photopeak or full absorption events in the observed spectrum is $\gamma p$ and that the photopeak pulse height distribution is described by a pdf, $P(h)$, then the proportion of photopeak events, misclassified as scatter will be given by :

$$
\varepsilon_{P}=\gamma_{P} \int_{-\infty}^{T} P(h) d h
$$

Thus the total error, $\mathcal{E}=\mathcal{E}_{\mathrm{P}}+\mathcal{E}_{\mathrm{C}}$. This can be minimized by differentiating, and setting to zero:

$$
\frac{\partial \varepsilon}{\partial h}=\gamma_{S} S(h)+\gamma_{P} P(h)=0
$$

At this point applying two Gaussian pdfs for $S(h)=N\left(\mu_{S}, \sigma_{S}\right)$ and $P(h)=N\left(\mu_{P}, \sigma_{P}\right)$ to equation 7 , after some manipulation, we arrive at a quadratic for $T$ : 


$$
\begin{aligned}
& \left(\sigma_{P}-\sigma_{S}\right) T^{2}+2\left(\sigma_{P}^{2} \mu_{S}-\sigma_{S}^{2} \mu_{P}\right) T- \\
& \mu_{P}^{2} \sigma_{S}^{2}+\mu_{S}^{2} \sigma_{P}^{2}-2 \sigma_{S}^{2} \sigma_{P}^{2} \ln \left(\frac{\sigma_{P}^{2} \gamma_{S}}{\sigma_{S}^{2} \gamma_{P}}\right)=0
\end{aligned}
$$

the solution of which represents where the 2 pdfs coincide. This can be re-written as

$$
\begin{gathered}
\left(\sigma_{P}-\sigma_{S}\right) T^{2}+2\left(\sigma_{P}^{2} \mu_{S}-\sigma_{S}^{2} \mu_{P}\right) T- \\
\mu_{P}^{2} \sigma_{S}^{2}+\mu_{S}^{2} \sigma_{P}^{2}-2 \sigma_{S}^{2} \sigma_{P}^{2} \ln \left(\frac{w_{S}}{w_{P}}\right)=0
\end{gathered}
$$

where $w_{S}, w_{P}$ are the scaling factors applied to each Gaussian.

The scaling factors $w_{S}$ and $w_{p}$ presented in equation 11 need to be estimated. This can be achieved using two energy windows either side of the photopeak. The width of these two windows was chosen to be $4 \mathrm{keV}$. Then using the model the counts in each window, $\Sigma$, can be described by:

$$
\Sigma=w_{P}\left(\operatorname{erf}\left(P\left(h_{U}\right)\right)-\operatorname{erf}\left(P\left(h_{L}\right)\right)\right)+w_{S}\left(\operatorname{erf}\left(S\left(h_{U}\right)\right)-\operatorname{erf}\left(S\left(h_{L}\right)\right)\right)
$$

where $\operatorname{erf}(z)=\frac{2}{\sqrt{\pi}} \int_{0}^{t} \exp \left(-t^{2}\right) d t \quad$ and $h_{U}, h_{L}$ represent the upper and lower limits of the window.

Applying equation 10 to the two sub-windows in each experimentally obtained spectrum, two equations are created with two unknowns $\left(w_{P}\right.$ and $\left.w_{S}\right)$ which can be solved.

The final step is to calculate the energy threshold by solving the quadratic in equation 9. We take the lower valued solution as the optimal lower energy threshold. The higher valued solution could then be used to define the upper window threshold. However, this may conflict with hardware implemented pulse pile-up rejection, so this has not been investigated in any detail.

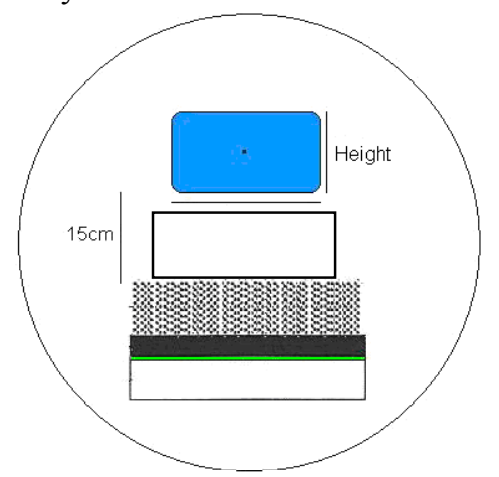

Figure 2. Side view geometry used for the simulation work. Same distance from the collimator was used for all the phantoms.

\section{Monte CARlo Simulation}

Knowing that there is no absolute point in which the Compton component distinguishes itself from the photopeak in Tc-99m studies, MCNP-based Monte Carlo simulations were undertaken to examine the Compton spectrum. The geometry used represented a Toshiba GCA-16000 Gamma camera imaging the projected flux from the flat face of a cylindrical phantom filled with water as a scattering medium and containing a Tc-99m point source at its center as shown in Fig. 2. The simulation tracks photons through the phantom, the collimator and the scintillation crystal. A PMT backscattercompartment is also included as part of the camera simulation. Energy dependent blurring was included based on a-priori experimentation on a real Toshiba camera, along with statistically sampled spatial blurring corresponding to a $\mathrm{FWHM}=3.5 \mathrm{~mm}$. Further details of this simulation appear in [12]. This allowed us to separate the Compton from the unscattered spectral components. Fig. 3 illustrates an exemplar component spectrum

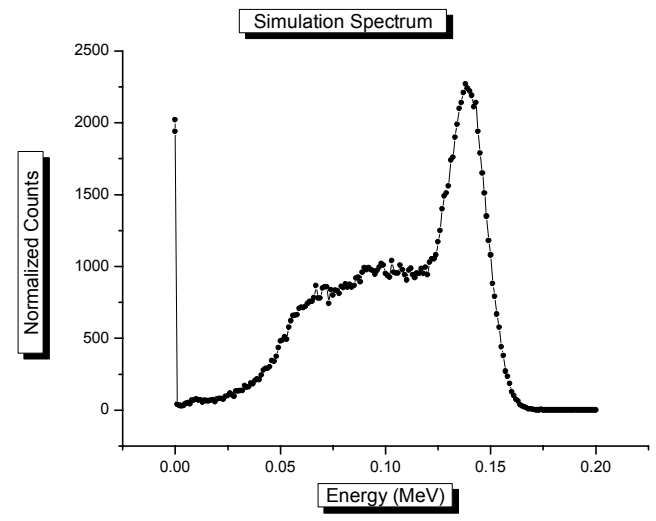

Fig. 3 Energy spectrum obtained from simulation

In order to fit a Gaussian distribution function to the Compton edge, an exhaustive search was conducted to determine the minimum Mean Square Error (MSE). MSE values were calculated as the difference between the simulated spectrum, $s$, and the estimated Gaussian distribution function $G_{C}\left(\mu_{c}, \sigma_{c}\right)$. Thus:

$$
M S E=\sum_{\mu=110}^{130} \sum_{\sigma=10}^{30} \frac{\left(s-G_{C}\left(\mu_{c}, \sigma_{c}\right)\right)^{2}}{N}
$$

This empirical Gaussian model is assumed to be valid for values of $\mu$ above $110 \mathrm{keV}$. The values of $\sigma$ are varied between 10 and 30 .

Fig. 4 presents exemplar MSE error surfaces, whilst Fig. 5 shows an excellent fit of the two compartments to a simulated spectrum. We conclude that this suggests that a simple Gaussian model is able to model the observed energy spectrum for photon energies $>120 \mathrm{keV}$. We then varied the volume of the simulated water filled cylinder and compared the true optimal threshold, observed by separating the energy blurred Compton events from the photopeak events with that obtained from using the GMM described above, as shown in 
Fig. 6. This shows excellent agreement between the two, illustrating the expected rise in optimal threshold with phantom volume. It is worth noting that with this particular geometry, a standard $20 \%$ window with a lower level threshold of $126 \mathrm{keV}$ would only be optimal for a phantom volume of $700 \mathrm{cc}$. Using such a lower threshold for larger volumes produces a greater scatter fraction than might otherwise be desirable.

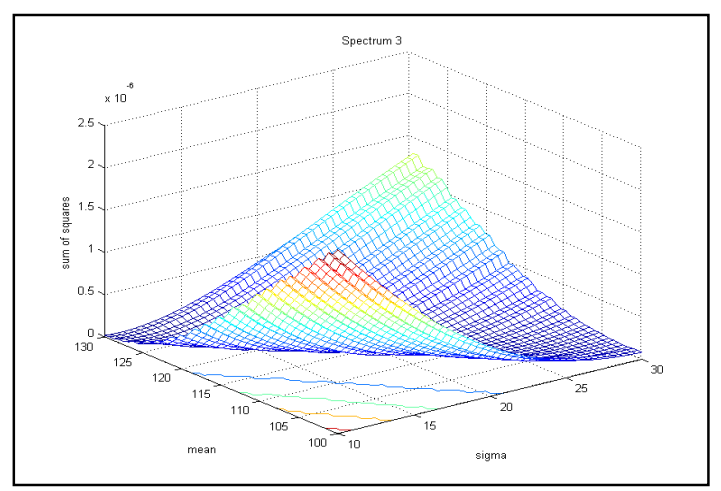

Fig. 4 Exemplar MSE error surfaces,

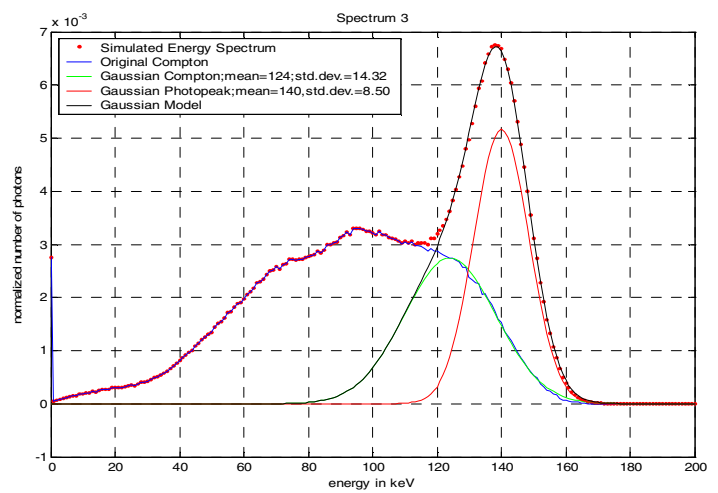

Fig. 5 Simulated spectrum with Gaussian fits to photopeak and Compton edge. Similar levels of fitting were observed for a variety of scattering volumes from which Fig. 6 was derived. Blue line: Compton component. Green and red lines: two components from the 2 class Gaussian model. Note excellent model agreement with spectra for photon energies $>120 \mathrm{keV}$.

\section{PReliminary Phantom StUdy}

Experimental work was carried out using an Infinia $3 / 8$ " or 1" Dual-Detector Cameras Hawkeye Hybrid NM/CT by GE Medical Systems.

A SPECT acquisition was undertaken, imaging a water filled phantom containing $330 \mathrm{MBq} \mathrm{Tc}-99 \mathrm{~m}$ and containing a cold spherical Perspex insert. First this was acquired with a $20 \%$ window, and then a second acquisition made where the lower threshold was changed to the optimal threshold predicted by the GMM Bayes' model. The phantom was placed on the gamma camera couch and the two gamma camera heads were rotated around it. A full $360^{\circ}$ scan of the phantom was accomplished with $20^{\circ}$ steps up to $180^{\circ}$ displacement from the starting position. Both detectors were at a distance of approximately $22 \mathrm{~cm}$ from the center of the cylinder phantom. The count profile through the reconstructed cold insert and further experimental details are shown in Fig. 7 This demonstrates that when using the window with the optimal energy threshold value, the non-radioactive sphere is more accurately represented by a lower gray level value than that obtained when using a standard $20 \%$ energy window. A bigger sphere would probably have increased the effect since in this case the sphere is relatively small $(9.5 \mathrm{~mm}$ in diameter $)$ in comparison with the size of the phantom $\left(7600 \mathrm{~cm}^{3}, 22 \mathrm{~cm}\right.$ in diameter).

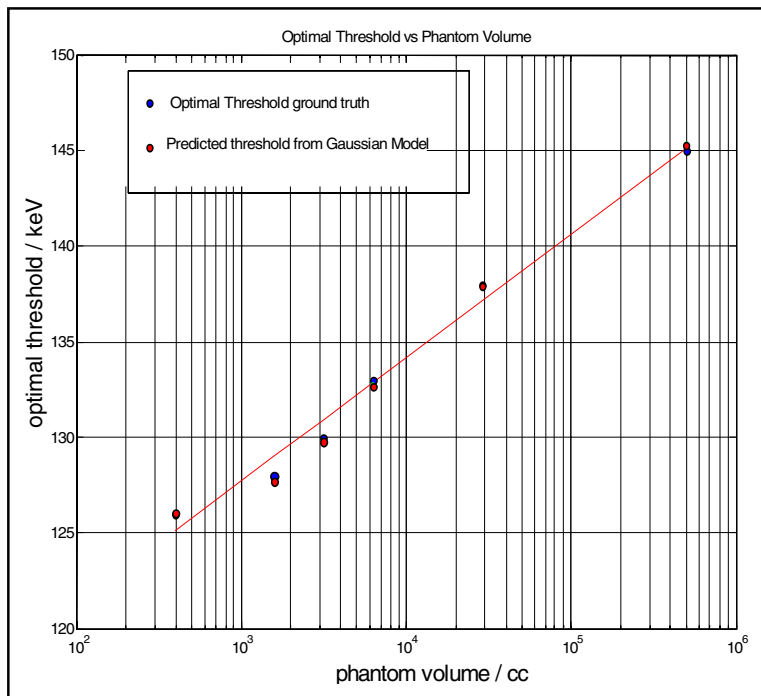

Fig. 6 Optimal threshold as a function of phantom volume for a cold object with a point source at the center. Insignificant variation observed between 'true' optimal window threshold define by simulation and the fitted optimal window set using the method described here.

\section{CONCLUSIONS}

This work has demonstrated that the Compton edge may be empirically represented by a Gaussian distribution function over a wide range of object scattering volumes. This has allowed the upper part of the energy spectrum to be well described by a 2-class Gaussian mixture model.

Having robustly modeled the energy spectrum, we have then used a Bayes' Minimum Error Threshold approach to set an optimal threshold. Preliminary experimental data has shown that this can improve image contrast in the presence of scatter for cold spot imaging. 


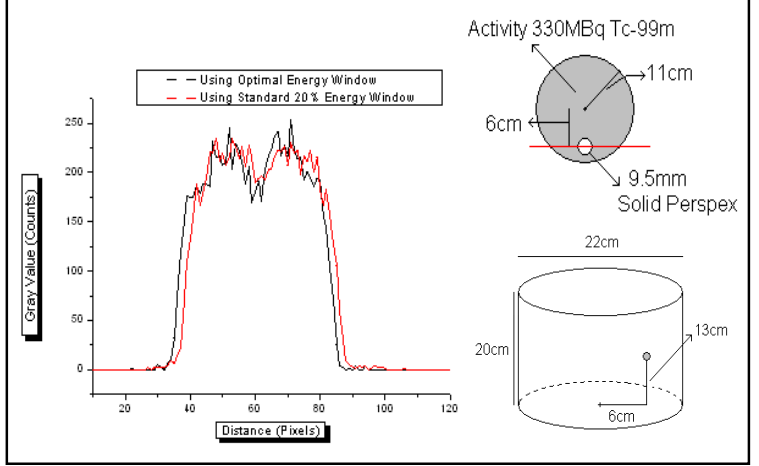

Fig. 7 Count Profile from a SPECT scan of a $22 \mathrm{~cm}$ diameter $\mathrm{x} 20 \mathrm{~cm}$ cylinder filled with $333 \mathrm{MBq}$ Tc- $99 \mathrm{~m}$ containing a $9.5 \mathrm{~cm}$ solid Perspex insert (right) using the GE HawkEye camera system (attenuation, but no scatter correction, applied). Inset: the imaging geometry. Red line indicates the location of the profile. Using an optimal threshold gives better contrast compared to a standard $20 \%$ window.

\section{ACKNOWLEDGMENT}

This work has been supported by a pump priming research award from the Royal Surrey County Hospital NHS Trust.

\section{REFERENCES}

[1] F. B.Atkins et al (1977) in Medical Radionuclide Imaging; Vol 1; Vienna IAEA pp 101-118

[2] F.D. Rollo (1974) Journal of Nuclear Medicine; Vol 15, pp 757 - 762

[3] P. Bloch \& T. Sanders (1973) Journal of Nuclear Medicine; Vol.14, pp 6772

[4] K. F. Koral et al (1986) NIM A242; pp 610-616

[5] L. S. Graham et al (1986) Journal of Nuclear Medicine; Vol 27; pp 706-713

[6] T.G. Turkington et al (2004) 'Adjusting the low energy threshold for large bodies in PET' IEEE NSS-MIC Conference Proceedings, Rome October 2004.

[7] T.G. Turkington et al (2005) IEEE NSS-MIC Conference Proceedings, Puerto Rico October 2005.

[8] I. Buvat, H. Benali, A. Todd-Pokropek and R. Di Paola, Scatter Correction in Scintigraphy: The State of the Art, European Journal of Nuclear Medicine, 21:675-694, 1994.

[9] M.J. Berger and S.M. Seltzer (1972), Nuclear Instruments and Methods, 104:317-332

[10] M. Sonka, V. Hlavac, R. Boyle, (1999) Image Processing Analysis and Computer Vision; $2^{\text {nd }}$ Edition International Thomson Publishing; pp128 - 129 and references therein.

[11] M. Petrou \& P. Bosdoggiani; (1999) Image Processing: the Fundamentals; Wiley \& Sons

[12] M. I Saripan et al; Medical Image Understanding \& Anlysis Conference Proceedings (2005) Bristol, UK

[13] A. Tumian (2004) 'Optimal Thresholding in Nuclear Medicine' MSc Dissertation, University of Surrey 